Research Article

\title{
Research on Fault Feature Extraction of Hydropower Units Based on Adaptive Stochastic Resonance and Fourier Decomposition Method
}

\author{
Yan Ren $\mathbb{D}^{1},{ }^{1,2}$ Jin Huang $\mathbb{C}^{1},{ }^{1}$ Lei-Ming Hu, ${ }^{3}$ Hong-Ping Chen, ${ }^{4}$ and Xiao-Kai Li ${ }^{4}$ \\ ${ }^{1}$ School of Electric Power, North China University of Water Resources and Electric Power, Zhengzhou 450045, China \\ ${ }^{2}$ Hunan Key Laboratory of Renewable Energy Power Technology, Changsha University of Science and Technology, \\ Changsha 410114, China \\ ${ }^{3}$ Jiang Xi Hong Ping Pumped Storage Company Limited, Nanchang 330000, China \\ ${ }^{4}$ State Grid Hunan Electric Power Company Limited, Changsha 410004, China \\ Correspondence should be addressed to Jin Huang; 1220311367@qq.com
}

Received 15 November 2020; Revised 21 December 2020; Accepted 16 February 2021; Published 1 March 2021

Academic Editor: Ling Zhou

Copyright $\odot 2021$ Yan Ren et al. This is an open access article distributed under the Creative Commons Attribution License, which permits unrestricted use, distribution, and reproduction in any medium, provided the original work is properly cited.

\begin{abstract}
In order to effectively extract the characteristics of nonstationary vibration signals from hydropower units under noise interference, an adaptive stochastic resonance and Fourier decomposition method (FDM) based on genetic algorithm (GA) are proposed in this paper. Firstly, GA is used to optimize the resonance parameters so that the signal can reach the optimal resonance and the signal-to-noise ratio (SNR) can be improved. Secondly, FDM is used to process the signal and the appropriate frequency band function is selected for reconstruction. Finally, Hilbert envelope demodulation analysis was performed on the reconstructed signal to obtain the fault characteristics from the envelope spectrum. In order to prove the effectiveness and superiority of the proposed method, comparative experiments are designed by using the simulated signal and the measured swing signal of a hydropower unit. The results show that this method can effectively remove the noise interference and improve the SNR and extract the characteristic frequency of the signal, which has the extensive engineering application value to the fault diagnosis of hydropower units.
\end{abstract}

\section{Introduction}

Hydropower has been playing an important role in the supply of energy due to its advantages of being clean and environmentally friendly, not consuming fossil fuels, and flexible operation. Hydropower units are the core components in power station operation $[1,2]$, and their safe and stable operation is of great significance to the benefits of a power station and even the stability of an entire power system. In order to ensure the stable operation of hydropower units, it is necessary to evaluate the operating status of hydropower units timely and effectively. The status assessment of hydropower units specifically includes signal acquisition, signal preprocessing, feature extraction, and fault diagnosis, among which feature extraction is the most critical step [3]. Research shows that more than $70 \%$ of hydropower unit faults are reflected in the form of vibration. Therefore, fault analysis and feature extraction methods based on vibration signals are crucial for improving the safety of hydropower units and extending the life of equipment. However, due to the complexity in the operating condition of hydropower units, the vibration signals collected contain not only environmental noise interference but also mixed fault information. From the perspective of vibration source, the fault information can be divided into three aspects: hydraulic factor, mechanical factor, and electromagnetic factor. Therefore, it is worth studying how to extract the weak characteristics of the early faults of hydropower units from the complex operating conditions.

Due to the complexity of unit structures and operating environment, the vibration signals collected often show 
strong nonstationary and time-varying characteristics, which make it difficult to extract the fault characteristics of units under the background of noise. Moreover, although the hydrodynamics of hydraulic machinery has been widely reported [4-7], the related diagnostic methods are seldom studied [8].On this basis, many time-frequency analysis methods have been proposed to extract signal characteristics. In these methods, EMD is a new adaptive decomposition method proposed in [9], which can adaptively decompose nonlinear and nonstationary signals without being disturbed by complex environment $[10,11]$. Therefore, it has been widely applied in various fields of engineering. However, this method is prone to mode mixing during the decomposition process, resulting in the failure of signal decomposition and the loss of useful frequencies. In order to solve the problem, scholars have successively proposed some modified versions of the algorithm such as EEMD $[12,13]$ and CEEMD $[14,15]$. In order to extract mechanical fault features effectively, a fault feature extraction method combining EEMD and multiscale fuzzy entropy was proposed and applied to motor bearing vibration signals so as to accurately diagnose the fault type and severity of inner ring faults [16]. For the sake of improvement in the effectiveness of early identification of rolling bearing faults, a rolling bearing fault diagnosis method combining CEEMD and correlation theory was proposed and utilized for the vibration signal collected from the rolling bearing fault test [17]. The results showed that the presented method can accurately detect the abnormal state of bearings in the early stage of operation and has high computational efficiency. However, the above modified algorithms not only add white noise to solve the mode mixing but also lead to white noise residue, which deteriorates the decomposition effect of signals. Fourier decomposition method (FDM) is an adaptive decomposition method based on the theory of the Fourier transform, in which the mode mixing will not appear in that extreme value points are not required. Moreover, due to that white noises are not added in signals, there is no residual of white noises [18-20]. In order to solve the difficulty in extracting fault characteristics of rotating machinery under the environment with a lot of noise, a method combining FDM, robust independent component analysis (RICA), and multipoint optimal minimum entropy deconvolution adjustment (MOMEDA) is proposed and applied to the analysis of actual bearing fault signal, and the effectiveness of suppressing noise and accurately extracting fault characteristic information is obtained, which shows its practicability in the fault diagnosis of rotating machinery [21].

For the sake of eliminating the noise interferences in the feature extraction of the weak fault signals of hydropower units, various signal processing methods to eliminate noise are developed to analyze the fault signal, such as intrinsic time-scale decomposition (ITD) [22], wavelet thresholdingbased denoising (WTD) [23, 24], and singular value decomposition (SVD) [25, 26]. Gang et al. [27] combined ITD and neural network to conduct fault diagnosis for rotating machinery vibration signals, where the anterior cruciate ligament (ACL) signals are first decomposed by ITD and then classified using a neural network. The results showed that the method can be used as the potential auxiliary tool for defects automatic detection of ACL in the clinical application. Fan and Zhu [28] used EMD and SVD to process vibration signals and achieved a satisfied effect in removing noise interference. Considering the unstable operating condition in industrial applications and the noise, Li et al. [29] put forward a new method for planetary gear box fault diagnosis, using the singular value decomposition (SVD) to enhance the resolution of the TFD obtained by the Wigner-Ville distribution (WVD). The results showed that the proposed method can not only reduce the influence of the cross terms of WVD but also eliminate noise and improve the accuracy of fault diagnosis. Unlike the conventional denosing methods, stochastic resonance (SR) is a method that reduces noise interference by transferring noise energy to fault signals. Through using a nonlinear system, part of the noise energy is transferred to the low-frequency signal, and the resonance of the weak signal submerged in the noise is strengthened so as to reduce the noise interference and extract the signal features effectively [30-32]. In order to solve the feature extraction and fault diagnosis of rolling bearings under a large amount of noise, Li and Shi [33] proposed a signal processing method that combined EEMD and adaptive stochastic resonance and applied it to the rolling bearing vibration signal, effectively enhancing the weak fault features and extracting them. Considering that the weak fault characteristics of mechanical equipment are usually difficult to be extracted from strong noise, the new SR method that utilized the classical potential energy is applied to the fault vibration signal of rotating machinery, achieving an effective fault feature extraction for the analog signal with heavy noise [34].

Based on the abovementioned analysis, considering the environmental noise interference during early weak fault feature extraction, a feature extraction method for hydropower unit vibration signal based on optimal SR and FDM is proposed in this paper to realize the accurate feature extraction for early weak fault, which is beneficial for the result of subsequent fault diagnosis. Firstly, the original vibration signals are processed by stochastic resonance with the system optimized by genetic algorithm, which strengthens the weak characteristic frequency component of the fault signal of the hydropower units. Then, FDM is used to adaptively decompose the resonant fault enhancement signal, and a series of modal functions are obtained. Next, the spectrum diagram of each modal function is therefore computed, respectively, and the fault frequency is determined through observation. Finally, the frequency calculated by the spectrum diagram is compared with that calculated by empirical formula, and the fault frequency contained in the spectrum diagram is found so as to complete the fault identification and diagnosis.

\section{Adaptive Stochastic Resonance Based on Genetic Algorithm}

By adjusting the parameters $a$ and $b$ of the first-order nonlinear system to achieve the optimal match between the system, the noise, and the periodic input signal, a stochastic resonance can be generated. Through the stochastic 
resonance, the energy of the noise signal is transferred to the input signal, realizing the detection of weak fault signals with strong noise.

2.1. Stochastic Resonance System. For a stochastic resonance system, the most fundamental components are the input signal (e.g., periodic signal) and the noise (e.g., white noise). Generally, the input signal and noise are integrated to obtain the mixed signal, which is then processed by the nonlinear system, so as to obtain the output signal with enhanced SNR.

A nonlinear system commonly used for stochastic resonance is a nonlinear bistable system, whose Langevin equation is presented as follows:

$$
\frac{\mathrm{d} x}{\mathrm{~d} t}=-U^{\prime}(x)+f(t)+\Gamma(t) .
$$

Where $U(x)$ is the potential function, $U(x)=-\left(a x^{2} / 2\right)$ $+\left(b x^{4} / 4\right)$, and $a$ and $b$ are the system parameters, while $f(t)$ is the input signal, $\Gamma(t)$ is the input noise signal, and $x$ is the output signal of the system.

For a stochastic resonance system to produce resonance phenomenon, the input signal needs to meet the adiabatic approximation theory; specifically, the input signal frequency, amplitude, and noise intensity should be less than 1 . However, the vibration signals to be analyzed, which are collected in the practical application of rotating machinery engineering, are generally signals with high frequency (large parameters), where stochastic resonance processing cannot be directly applied to the actual signals. Therefore, a variablescale processing is first proposed to process the vibration signals to be analyzed. The basic principle of the method is to compress the linear signal by setting the compression ratio, so that the signal can meet the small parameter conditions to generate stochastic resonance phenomenon. Finally, the signal after resonance processing is restored to the original signal by scale transform.

The main steps in the process are as follows: a frequency compression ratio $R$ is first defined and the compressed sampling frequency is defined as $f_{s r}=(f / R)$ according to the frequency compression ratio. The timeline is redefined as $t^{\prime}=R t$. The step length and the driving frequency of the signal are set to $h^{\prime}=\left(1 / f_{s r}\right)$ and $f^{\prime}=(f / R)$, respectively. Finally, the output signal of stochastic resonance is solved according to the fourthorder Runge-Kutta algorithm and then is restored based on scale transform. Since the measured signal is compressed according to a certain frequency and not changed in its any property, the frequency characteristic analysis is not affected for the signal.

\subsection{Genetic Algorithm (GA) Optimized Adaptive Stochastic} Resonance System. Since the values of the parameters $a$ and $b$ in equation (1) and the noise intensity can affect the SNR and thus determine the denoising effect of the resonance system, one can deal with the problem from the system parameter and noise intensity to find the optimal SNR. In practical engineering applications, the noise intensity is generally determined and cannot be changed. Therefore, the optimal solutions of $a$ and $b$ are usually adopted to ensure that the SNR reaches the maximum value and the resonance system can achieve the optimal resonance effect.

Genetic algorithm (GA) is an algorithm that simulates evolutionary processes in nature to search for optimal solutions. The basic principle is that by constructing an objective function as fitness function and using genetic operators (selection, crossover, and mutation), a subsequent generation is selected based on the principle of "survival of the fittest," producing individuals with high fitness. After several generations of evolution, the fitness of population gradually enhanced, finally converging to an optimal solution. GA is now widely used in engineering because of its robustness and implicit parallelism.

In this paper, GA is applied to the parameter optimization for stochastic resonance systems. Based on the multiparameter optimization ability of GA and the use of the output SNR as fitness function, the parameters of stochastic resonance system, i.e., $a$ and $b$, are optimized, and then the best parameters are found. In this manner, the best resonance between the input signal and the noise is formed and the best SNR is obtained. .

The optimization steps of GA are as follows:

(1) Binary coding:

The aim of binary coding is to transform the feasible solution of optimization problem from solution space to search space that GA can handle. The search ranges of system parameters are set as follows:

$a \varepsilon\left[A_{\min }, A_{\max }\right], b \in\left[B_{\min }, B_{\max }\right]$, Then, according to equations (2) and (3), the corresponding code length $l$ and $k$ of the parameters are determined as

$$
\begin{aligned}
& 2^{l}-1=\frac{A_{\max }-A_{\min }}{\delta}, \\
& 2^{k}-1=\frac{B_{\max }-B_{\min }}{\delta},
\end{aligned}
$$

where $\delta$ is the accuracy that the code can represent. Then, perform binary coding, and get the codes corresponding to the individual as $c_{l} c_{l-1}, \ldots, c_{1}$, $d_{k} d_{k-1}, \ldots, d_{1}$. The corresponding system parameters can be obtained according to the decoding formula:

$$
\begin{aligned}
& a=A_{\min }+\left(\sum_{i=1}^{l} c_{i} 2_{i-1}\right) \frac{A_{\max }-A_{\min }}{2^{l}-1}, \\
& b=B_{\min }+\left(\sum_{i=1}^{k} d_{i} 2^{i-1}\right) \frac{B_{\max }-B_{\min }}{2^{k}-1} .
\end{aligned}
$$

(2) Initial population:

Set the population size and randomly select individuals to form the initial population. The higher the population size, the better the quality of the solution. However, if the population size is too large, the calculation cost will increase. Hence, it is usually chosen in a compromise in practical application. 
(3) Calculated fitness:

The selection of fitness function is the basis of algorithm optimization. Here, SNR is selected as the fitness function for GA. The system parameters corresponding to each individual are put into the stochastic resonance system, and the fitness is therefore calculated by applying fourth-order Runge-Kutta algorithm.

(4) Genetic process:

Genetic evolution can be divided into three parts: selection, crossover, and mutation. The selection is based on the law of "survival of the fittest," making the individual with good fitness value involved in the next generation of the reproductive process, eliminating the individuals with worse fitness value.

"Crossover" is the process of exchanging and recombining parts of genes from two fathers to create a new individual. Mutation is the process of changing certain gene positions of an individual with a certain probability. Through the abovementioned genetic evolution process, a new population can be produced from the old.

(5) Termination condition:

The whole evolutionary process is terminated when it reaches to the maximum iterations, and then the current optimal solution is outputted, that is, the best system parameters are obtained.

\section{Fourier Decomposition Methods (FDMs)}

FDM is based on the premise of defining Fourier intrinsic band functions (FIBFs), adaptively searches for FIBF components in the Fourier frequency domain, and minimizes the number of decomposed components. Nonlinear nonstationary signals can be decomposed into FIBFs and a residual component using FDM. Unlike traditional adaptive time-frequency domain analysis methods, FDM has the attributes of orthogonality, locality, and adaptability and can effectively avoid end effect in the decomposition process and mode mixing in the decomposition results.

First, the FIBF component, denoted as $y_{i}(t)$, must meet the following conditions:

(1) FIBF is a zero mean function, that is,

$$
\int_{a}^{b} y_{i}(t) \mathrm{d} t=0
$$

(2) FIBFs satisfy orthogonality, namely,

$$
\int_{a}^{b} y_{i}(t) y_{j}(t) \mathrm{d} t=0, \quad i \neq j
$$

(3) The instantaneous frequency $a(t)$ and instantaneous amplitude $\left(\mathrm{d} \Phi_{i}(t) / \mathrm{d} t\right)$ of the analytical functions AFIBFs of FIBFs are always not less than 0, that is,

$$
\begin{aligned}
& y_{i}(t)+j \widehat{y}_{i}(t)=a_{i}(t) \exp \left(j \Phi_{i}(t)\right), \\
& a_{i}(t)\left(\frac{\mathrm{d} \Phi_{i(t)}}{\mathrm{d} t}\right) \geq 0 .
\end{aligned}
$$

Next, FIBF is searched by using the following two methods:

(1) LTH-FS algorithm (Fourier spectrum from low frequency to high frequency):

Step 1: For complex signal $x(n)$, Fourier transform is performed to obtain $X(k)$,

$$
X[k]=\operatorname{FFT}\{x(n)\} .
$$

Step 2: Let the $i$-th AFIBF component of the signal $x(t)$ be

$$
\begin{aligned}
\operatorname{AFIBF}_{i} & =\sum_{k=\left(N_{i}-1+1\right)}^{N_{i}} X[k] \exp \left(\frac{j 2 \pi k n}{N}\right) \\
& =a_{i}[n] \exp \left(j \varphi_{i}[n]\right) .
\end{aligned}
$$

In order to obtain the AFIBFs with the least number, the $i$-th AFIBF is obtained by cumulative increase starting from $N_{i-1}+1$ to $N_{i}$ that satisfied the condition, and the phase $\varphi_{i}[n]$ that meets the $i$-th AFIBF is a monotonically increasing function, that is,

$$
\omega_{i}[n]=\left(\frac{\varphi_{i}[n+1]-\varphi_{i}[n-1]}{2}\right) \geq 0 .
$$

Step 3: Calculate the residual component $r(n)$,

$$
r[n]=X[0]+X\left[\frac{N}{2}\right]+X\left[\frac{N}{2}\right](-1)^{n} .
$$

(2) HTL-FS algorithm (Fourier spectrum from high frequency to low frequency):

Step 1: For the complex signal $x(n)$, perform Fourier transform to obtain $X[k]$, namely,

$$
X[k]=\operatorname{FFT}\{x(n)\} .
$$

Step 2: Set the $i$-th AFIBF component of the signal to be $x(t)$,

$$
\begin{aligned}
\operatorname{AFIBF}_{i} & =\sum_{k=N_{i}}^{N_{i-1}-1} X[k] \exp \left(\frac{j 2 \pi k n}{N}\right) \\
& =a_{i}[n] \exp \left(j \varphi_{i}[n]\right) .
\end{aligned}
$$

In order to obtain the AFIBFs with the least number, the $i$-th AFIBF is obtained by the cumulative decrease starting from $N_{i}$ to $N_{i-1}-1$ that satisfied the condition, and the phase $\varphi_{i}[n]$ that meets the $i$-th AFIBF is a monotonically increasing function, that is, 


$$
\omega_{i}[n]=\left(\frac{\varphi_{i}[n+1]-\varphi_{i}[n-1]}{2}\right) \geq 0 .
$$

Step 3: Calculate the residual component $r(n)$,

$$
r[n]=X[0]+X\left[\frac{N}{2}\right](-1)^{n} .
$$

Finally, the signal is decomposed into a finite number of AFIBFs and a residual component, namely,

$$
x(t)=\sum_{i=1}^{M} y_{i}(t)+r(t)
$$

In the formula, $r(t)$ is the residual component.

\section{Vibration Signal Feature Extraction for Hydropower Units}

Under the condition of strong noise, the early fault signal of hydropower units is weak and the SNR is low, which brings challenges for fault diagnosis. In view of the abovementioned problems, a method combined with GA-SR-FDM is proposed for extracting fault features of hydropower units. The flowchart is shown in Figure 1. The specific steps are as follows:

(1) In order to meet the small parameter condition of $\mathrm{SR}$, subsampling frequency scale transform is conducted for the early fault signal $x(t)$.

(2) The objective function is set as the output SNR of the system and genetic algorithm is utilized to optimize the structural parameters of SR, and the optimal values $a_{0}$ and $b_{0}$ of the parameters can be obtained.

(3) $a_{0}$ and $b_{0}$ are substituted into the SR system for Runge-Kutta calculation, which increases the SNR of the vibration signals of hydropower units and then the signal obtained after GA-SR is recorded as $x_{1}(t)$.

(4) $x_{1}(t)$ is adaptively decomposed by FDM to obtain a number of FIBFs with different frequency bands and a residual component. The correlation coefficients are adopted as a reference index to select the FIBFs that meet the parameter requirements to reconstruct vibration signal, denoted as $x_{2}(t)$, of units.

(5) According to the empirical formula of hydropower unit faults [35], $x_{2}(t)$ is analyzed with Hilbert transform to determine fault features.

\section{Simulation Analysis}

Under complex operating conditions, the vibration signal of hydropower units has nonlinear and nonstationary characteristics. Before applying the GA-SR-FDM method to the actual vibration signal for fault feature extraction, the method is first verified by simulation analysis.

First, the vibration simulation signal is generated on the basis of the operating characteristics of hydropower units. The rotational speed of a real unit considered in this paper is $120 \mathrm{r} / \mathrm{min}$, and the rotational frequency is $2 \mathrm{~Hz}$. Since the

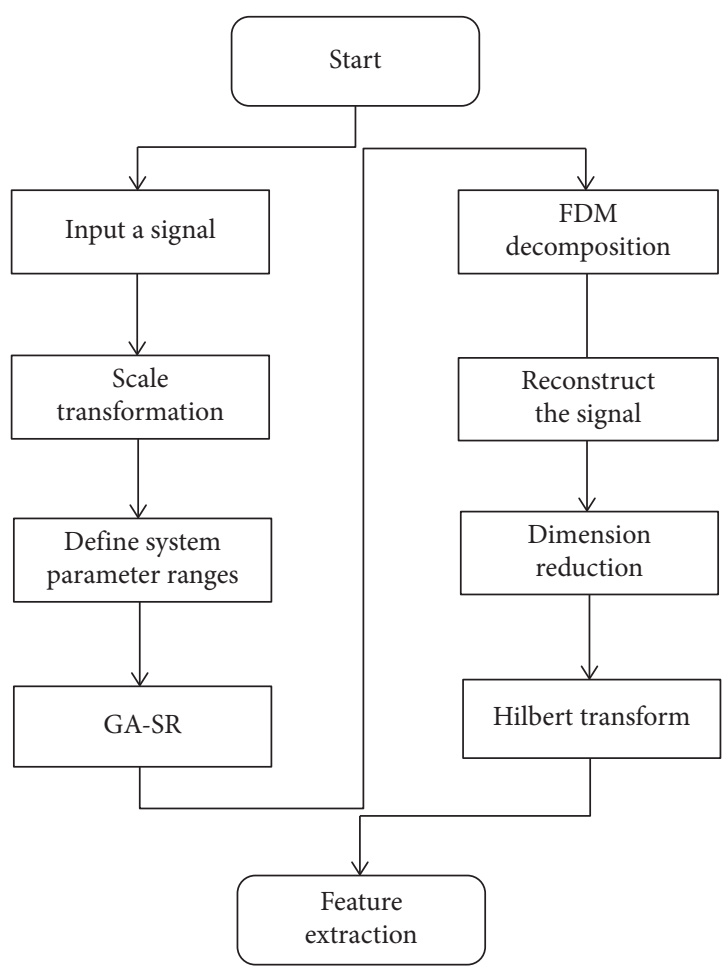

FIgURE 1: Flowchart of signal feature extraction for hydropower units.

early fault features of hydropower units are weak, usually including low-frequency components that are generally $1 /$ $5 \sim 1 / 3$ times rotational frequency and 5 times rotational frequency, the low frequency $0.008 \mathrm{~Hz}$ and $0.1 \mathrm{~Hz}$ is selected as the fault feature frequency to generate the simulation signal with noise, as shown in

$$
\left\{\begin{array}{l}
x^{s}=x_{1}^{s}+x_{2}^{s}, \\
x_{1}^{s}=0.01 \sin (2 \pi \times 0.008 \times t)+0.008 \sin (2 \pi \times 0.1 \times t) .
\end{array}\right.
$$

In equation (17), $x_{1}^{s}$ is a fault signal, $x_{2}^{s}$ is a noise signal that generates a noise set with the same length as that of $x_{1}^{s}$, and $t$ is time. The amplitude of the simulated signal is based on the actual signal.

The simulated signal $x^{s}$ to be processed is shown in Figure 2(a). It can be seen from the figure that the fault signal $x_{1}^{s}$ is drowned in the simulated noise signal $x_{2}^{s}$ due to its small amplitude, which cannot be clearly distinguished from either the waveforms or the frequency spectrum. In order to extract the fault feature from noise and prove the advantage of the proposed method, GA-SR, FDM, EMD, ITD, and GASR-FDM are applied to the simulation signal, respectively, and the results are shown in Figures 2(b)-2(f). As shown in the figure, there is a lot of noise frequencies mixed around the fault feature frequency after the signal is processed by the aforementioned methods. The feature frequency with $0.008 \mathrm{~Hz}$ and $0.1 \mathrm{~Hz}$ cannot be accurately extracted. Comparing the results among these methods, it is found that the feature frequency in the spectrum obtained by GA-SR-FDM is clear, without almost noise interference. Therefore, GA- 

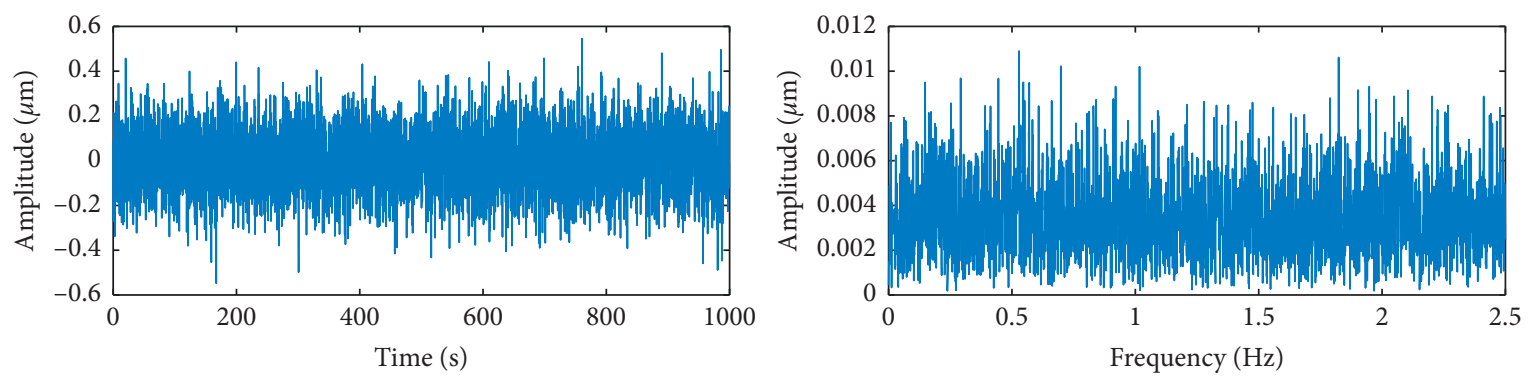

(a)
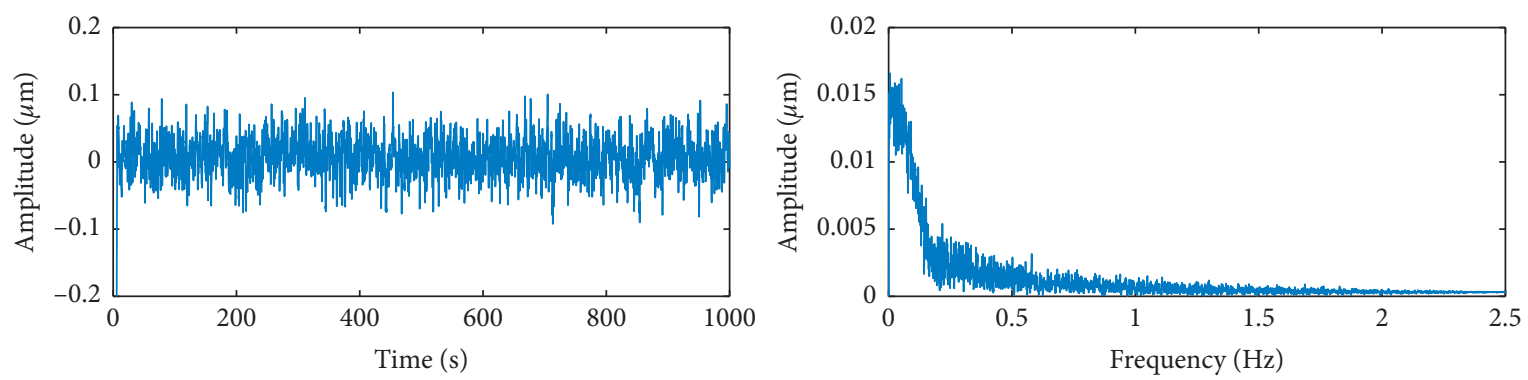

(b)
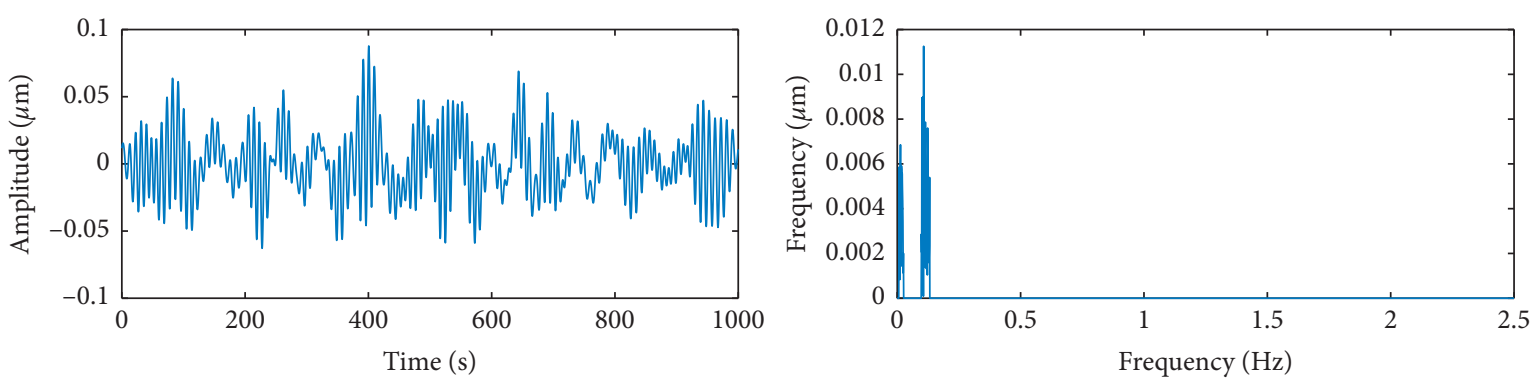

(c)
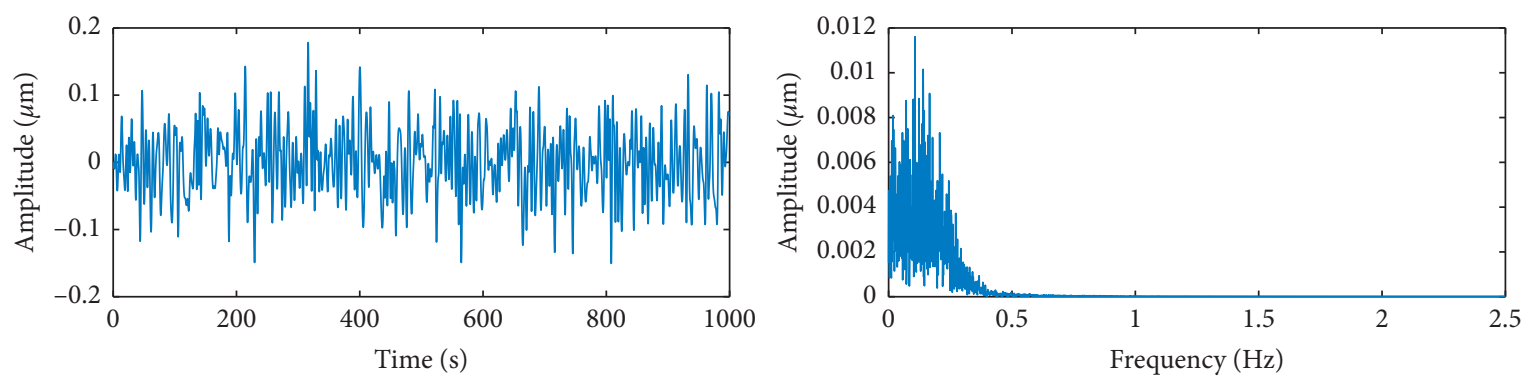

(d)
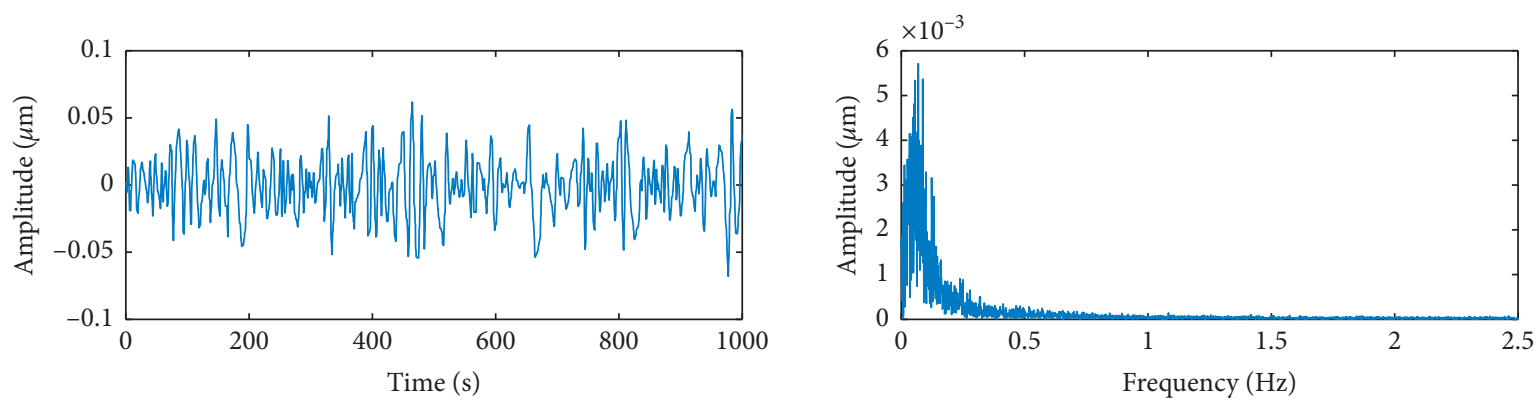

(e)

Figure 2: Continued. 

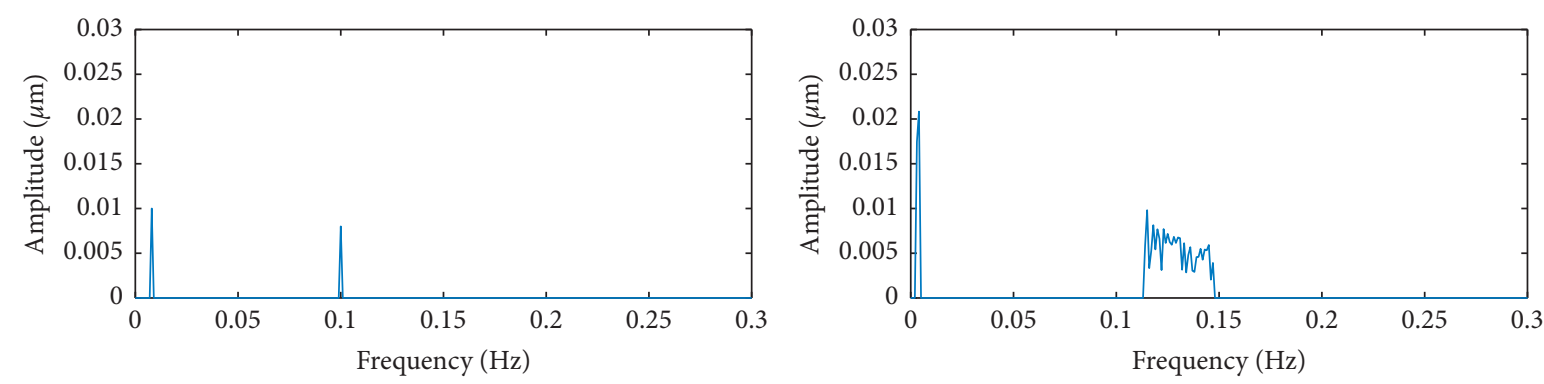

(f)

FIGURE 2: Waveform and frequency spectrum of the simulation signal processed by different methods. (a) Unprocessed simulated signal. (b) Simulated signal processed by GA-SR. (c) Simulated signal processed by FDM. (d) Simulated signal processed by EMD. (e) Simulated signal processed by ITD. (f) Fault signal and simulated signal processed by GA-SR-FDM.

Table 1: Performance comparison of each method.

\begin{tabular}{lccccc}
\hline & GA-SR & FDM & EMD & ITD & GA-SR-FDM \\
\hline SNR & -20.6740 & -7.9508 & -14.4336 & -7.8138 & -5.8816 \\
RMS & 1.5369 & 0.0226 & 0.0483 & 0.0223 & 0.0117 \\
\hline
\end{tabular}
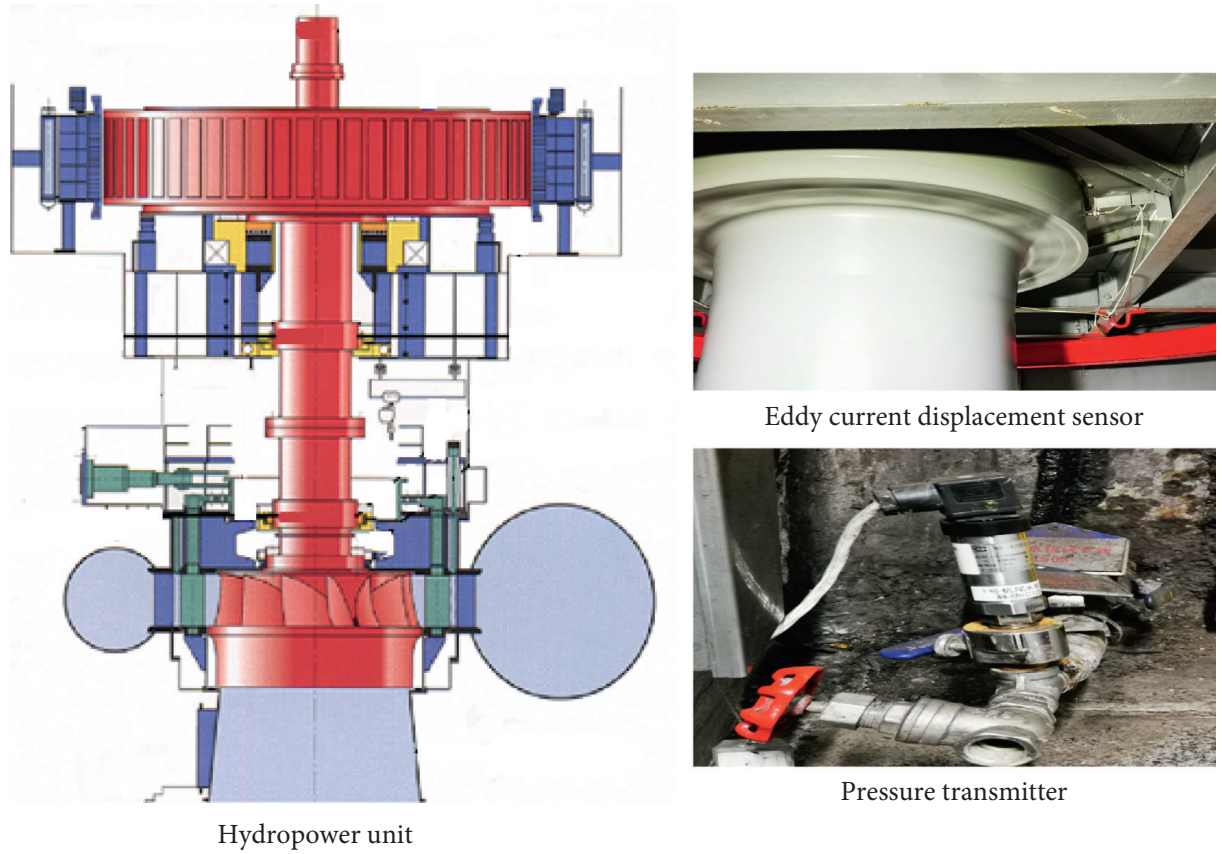

Eddy current displacement sensor

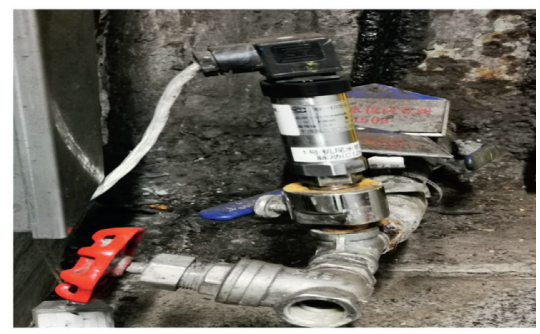

Pressure transmitter

FIgURE 3: Schematic diagram of the unit structure and sensor layout.

SR-FDM is more suitable for the feature extraction of weak fault signals than other methods.

In order to more scientifically reflect the performance of the aforementioned methods, the SNR and the root-meansquare (RMS) of the difference between the processed signal and original signal are calculated, as shown in Table 1. The SNR and RMS obtained by GA-SR-FDM are -5.8816 and 0.0117 , respectively, much lower than that of the other methods, which shows that the signal processed by the GASR-FDM contains less noise signals and more complete feature signals. Therefore, the proposed method can reduce noise interference and effectively extract weak fault features.

\section{Case Analysis}

A domestic hydropower unit is taken as an example to carry out the case analysis. The unit parameters are as follows: (1) the type of the hydro-turbine is HL-702-LJ-410; (2) the designed output power of the unit is $63 \mathrm{MW}$; (3) the rated speed $n$ is $136 \mathrm{r} / \mathrm{min}$; (4) the number of the unit blades $z_{0}$ is 14. The eddy current displacement sensors with the type of CWY-DO and the pressure transmitter with the type of AK$4 \mathrm{D}$ are installed at the flange and the draft tube, respectively. The layout of measured points is shown in Figure 3, where the sampling frequency is $500 \mathrm{~Hz}$. The vibration signal of 

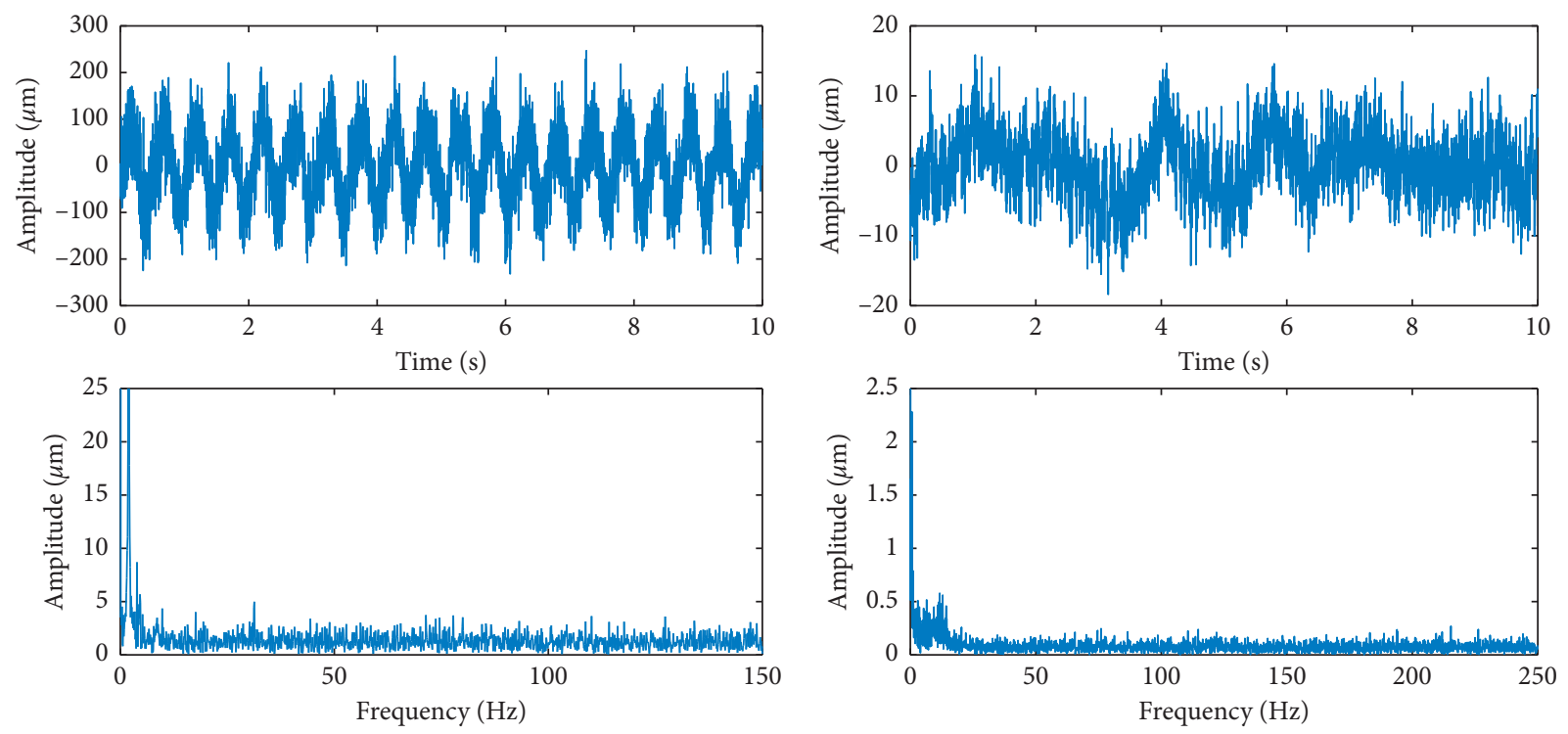

(a)

(b)

Figure 4: Actual signal of turbine. (a) Y-direction swing signal at the flange. (b) Vibration signal at the draft tube.
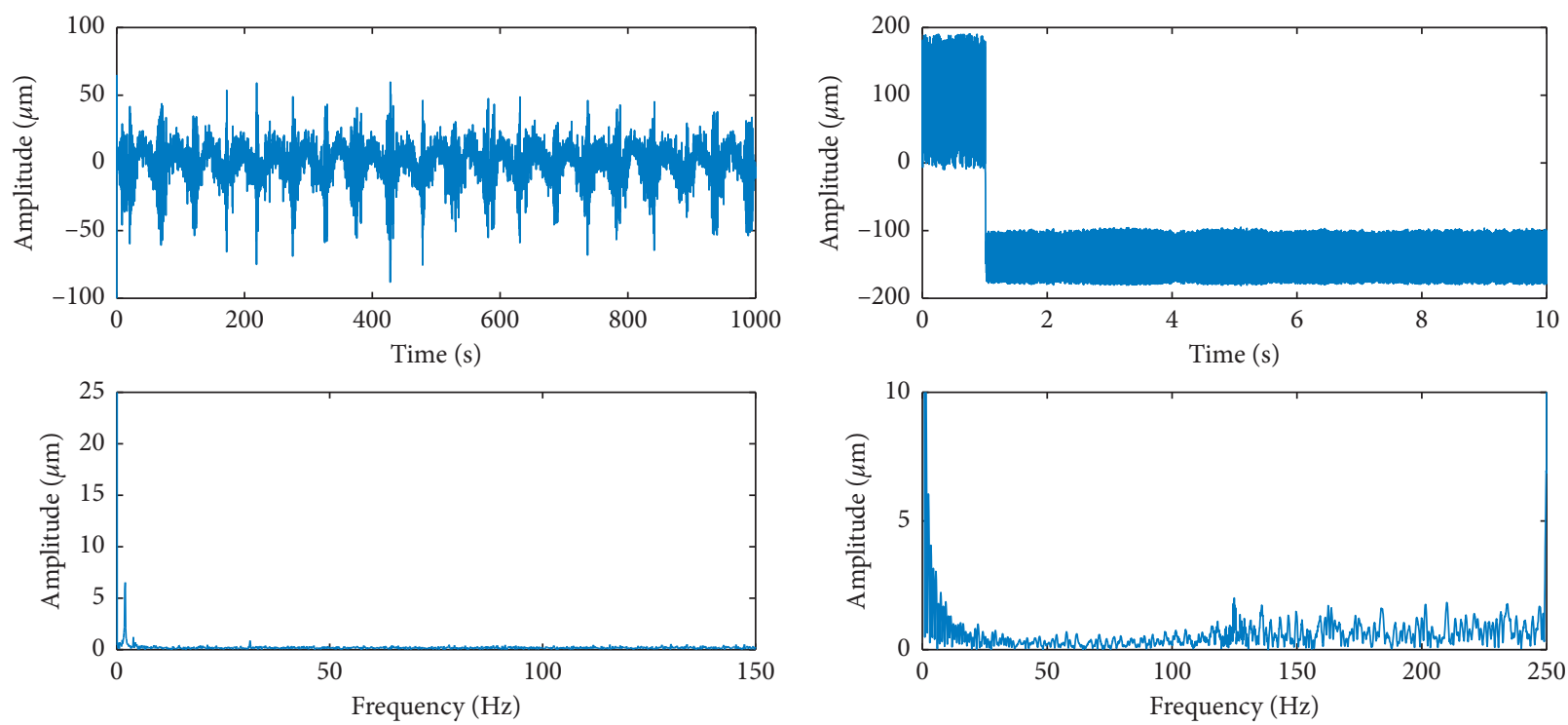

(a)

(b)

Figure 5: Actual signal processed by GA-SR. (a) Y-direction swing signal at the flange. (b) Vibration signal at the draft tube.

flange at horizontal direction and the pressure pulsation signal of the draft tube are adopted to analyze and to further verify the advantage of the proposed method.

The waveforms and the frequency spectrum of the original signal without being processed are shown in Figure 4 . The waveforms and the frequency spectrum of the original signal processed by aforementioned methods are shown in Figures 5-9.

In order to further compare the effect of feature extraction by aforementioned methods, feature frequencies of some typical faults are summarized as a reference, which is shown in Table 2 [35]. The unit rotational frequency $f_{0}$ can be calculated from the rated speed, namely, $2.26 \mathrm{~Hz}$.

As shown in Figure 4, due to the small amplitude, the feature frequencies of the fault analyzed are submerged in the noise signal and hard to be extracted from the waveforms and the frequency spectrum. Hence, it is difficult to judge whether a fault occurs.

The results obtained by GA-SR and FDM are shown in Figures 5 and 6, respectively. It can be seen that the feature frequency of the signal processed by GA-SR can be identified to a certain extent, indicating that although GA-SR can 

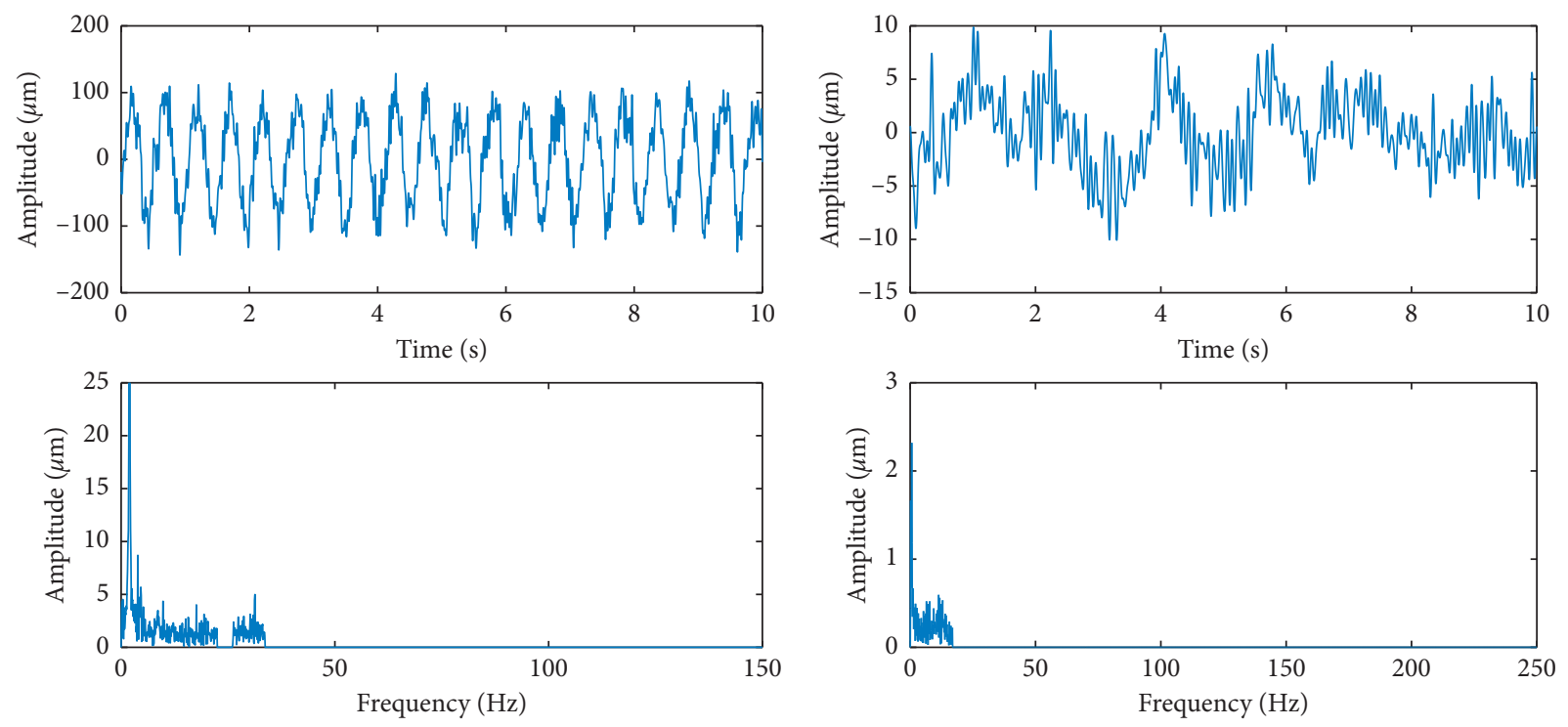

(a)

(b)

Figure 6: Actual signal processed by FDM. (a) Y-direction swing signal at the flange. (b) Vibration signal at the draft tube.
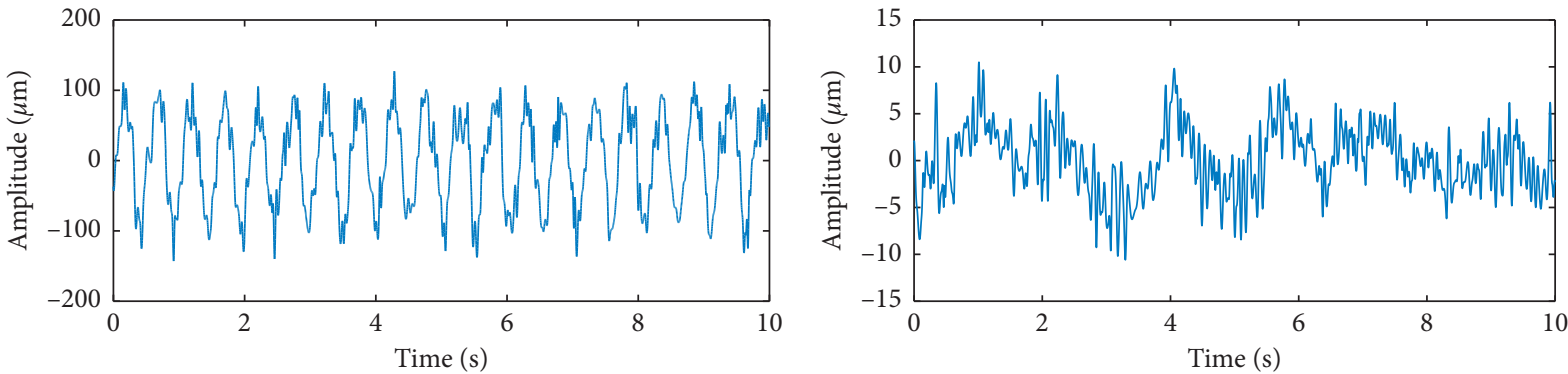

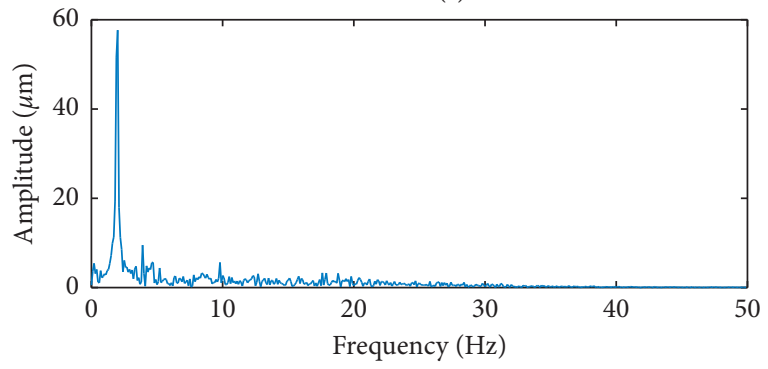

(a)

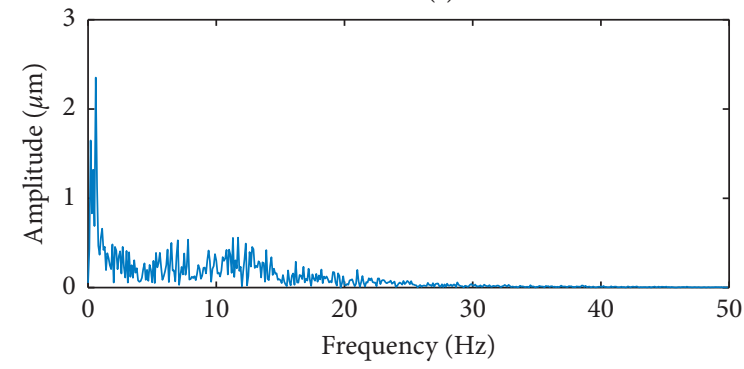

(b)

Figure 7: Actual signal processed by EMD. (a) Y-direction swing signal at the flange. (b) Vibration signal at the draft tube.

effectively enhance the amplitude of the feature frequency and the SNR, it cannot completely eliminate noise interference. After processed by FDM, the noise of the signal is partially removed, indicating that FDM can be used for feature extraction of nonlinear and nonstationary signals, but some useful information may be lost.

The results of EMD and ITD processing are shown in Figures 7 and 8 , respectively. It can be seen that the fault signals processed by EMD and ITD contain a lot of noise, which makes the fault feature frequency unable to be accurately extracted. This is because there is a certain mode mixing caused by EMD, while for ITD, the baseline signals are constructed by linear transformation, leading to glitch and distortion in processed signals, which reduces the effectiveness and reliability of diagnosis results.

Comparing Figure 6 with Figures 7 and 8, it is obvious that the signal processed by FDM has no mode mixing and has a certain fault feature frequency, which indicates FDM is 

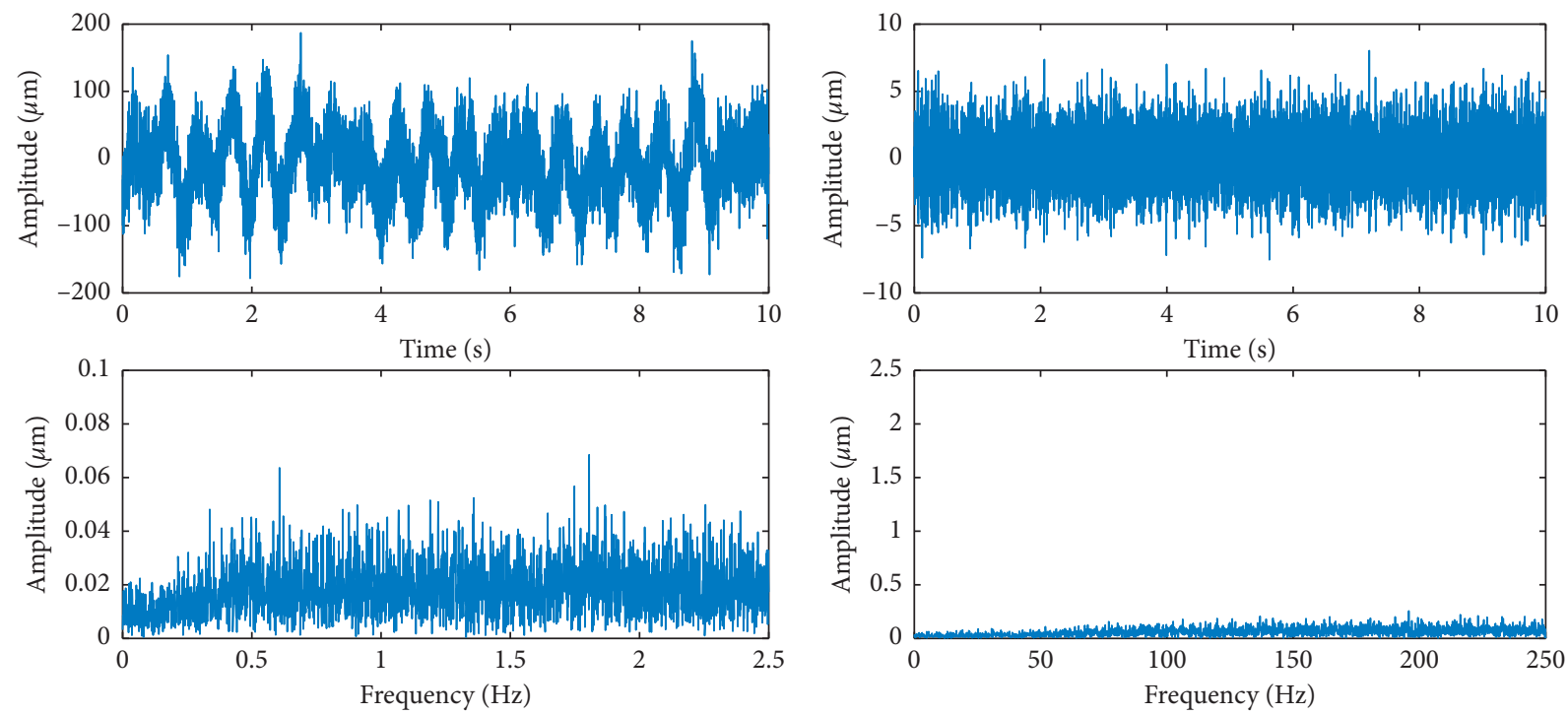

(a)

(b)

FIgURE 8: Actual signal processed by ITD. (a) Y-direction swing signal at the flange. (b) Vibration signal at the draft tube.
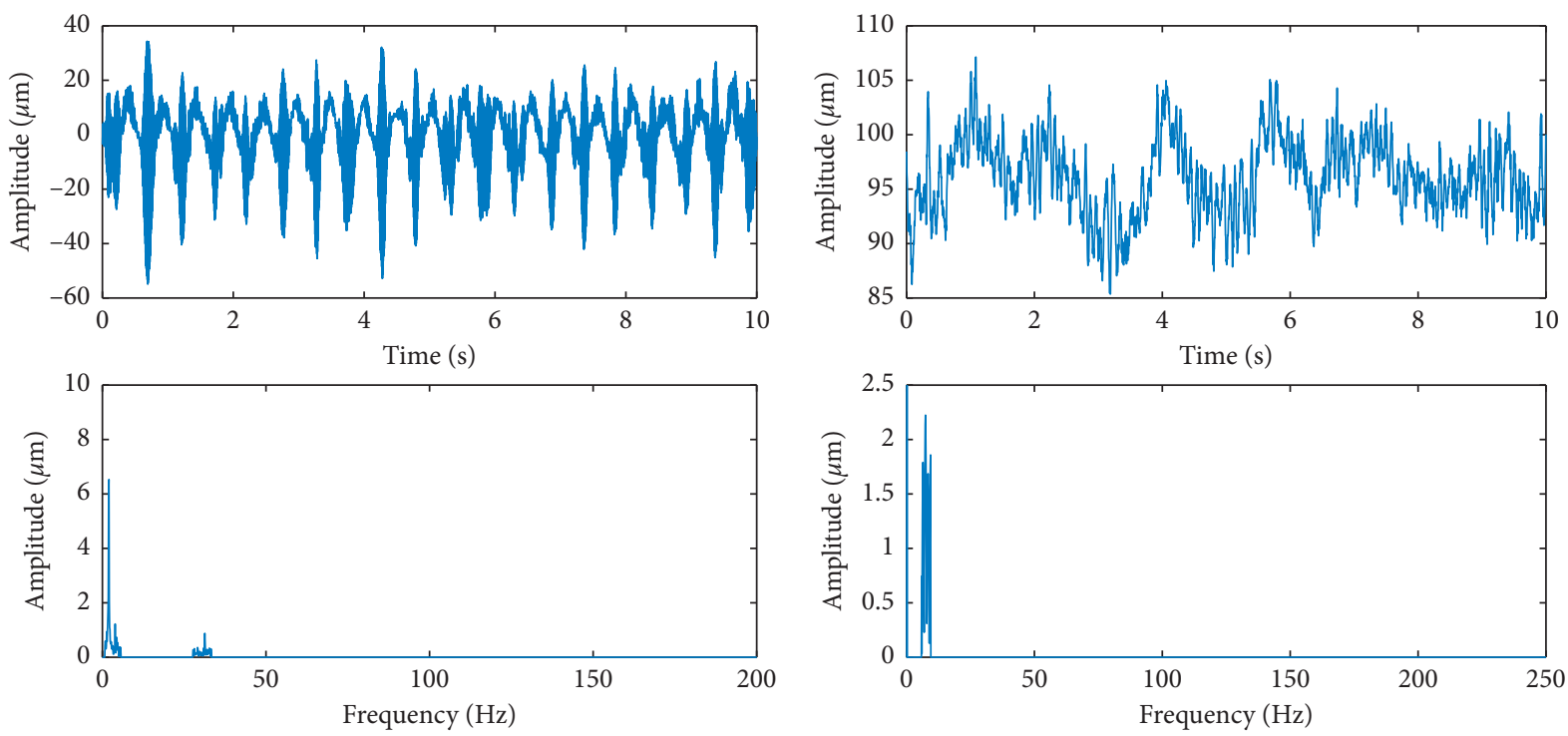

(a)

(b)

Figure 9: Actual signal processed by GA-SR-FDM. (a) Y-direction swing signal of the flange. (b) Signal at the draft tube.

TABLE 2: Partial vibration fault characteristics of hydropower units.

\begin{tabular}{lc}
\hline Feature frequency & Typical faults \\
\hline$f_{0}$ & Rotor mass imbalance; improper clearance of guide bearing \\
$(1 / 5) f_{0} \sim(1 / 2) f_{0}$ & Low-frequency pressure fluctuation of draft tube \\
$z_{0} f_{0}$ & Uneven opening of guide vane \\
\hline
\end{tabular}


more effective in extracting fault features under strong noise conditions than EMD and ITD.

The result of GA-SR-FDM processing is shown in Figure 9. As can be known, the amplitudes corresponding to frequency of $1 \mathrm{~Hz}, 2.26 \mathrm{~Hz}$, and $31.64 \mathrm{~Hz}$ are enhanced, thereafter the feature frequency is effectively extracted. As a result, compared to other processing methods, GA-SR-FDM can effectively remove the environmental noise and obtain a higher SNR, which indicates it is more suitable for feature extraction of early weak fault signals.

\section{Conclusion}

In this paper, a new method to extract the weak fault features of vibration signals under the background of strong noise is proposed for hydropower units. In order to prove the effectiveness and advantages of the proposed method, the simulation signal and the actual signals at different place of the hydropower unit is utilized and various comparative experiments are designed. Some conclusions are summarized as follows:

(1) FDM avoids the mode mixing in EMD and the glitch and distortion in ITD and can be used to extract the feature frequency of nonlinear and nonstationary signals.

(2) For the early weak fault signal of hydropower units mixed with strong noise, the modified SR in the proposed method transfers part of the noise energy to the low-frequency band of signals through a nonlinear system. Therefore, the amplitude and the SNR of the fault signal are increased, and the noise suppression is achieved.

(3) The traditional SR requires the system to meet the conditions of small parameter, while the vibration signals of the hydropower units belong to the large parameter signal. Therefore, the variable-scale signal processing methods are adopted to change the parameters of the vibration signal for the units to meet the conditions of SR.

(4) The traditional SR system cannot adaptively select the values of structural parameters (i.e., $a$ and $b$ in this paper). To solve the problem, GA is used in this paper to adaptively optimize the parameters so as to obtain stochastic resonance under the best parameter values.

(5) The GA-SR-FDM proposed in this paper has an obvious effect on the feature extraction of early weak fault signals of hydropower units under the condition of strong noise, which provides a theoretical basis for the fault diagnosis of hydropower units.

\section{Data Availability}

The data used to support the findings of this study are available from the corresponding author upon request.

\section{Conflicts of Interest}

The authors declare that they have no conflicts of interest.

\section{References}

[1] R. B. Randall and J. Antoni, "Rolling element bearing diagnostics-A tutorial," Mechanical Systems and Signal Processing, vol. 25, no. 2, pp. 485-520, 2011.

[2] L. Yaguo, L. Jing, H. Zhengjia, and J. Z. Ming, "A review on empirical mode decomposition in fault diagnosis of rotating machinery," Mechanical Systems and Signal Processing, vol. 35, no. 1-2, pp. 108-126, 2013.

[3] Z. Feng and F. Chu, "Nonstationary vibration signal analysis of a hydroturbine based on adaptive chirplet decomposition," Structural Health Monitoring, vol. 6, no. 4, pp. 265-279, 2007.

[4] H. Yan, X. Su, H. Zhang et al., "Design approach and hydrodynamic characteristics of a novel bionic airfoil," Ocean Engineering, vol. 216, Article ID 108076, 2020.

[5] Y. Yang, L. Zhou, W. Shi et al., "Interstage difference of pressure pulsation in a three-stage electrical submersible pump," Journal of Petroleum Science and Engineering, vol. 196, Article ID 107653, 2021.

[6] L. Zhou, C. Han, L. Bai et al., "CFD-DEM bidirectional coupling simulation and experimental investigation of particle ejections and energy conversion in a spouted bed," Energy, vol. 211, Article ID 118672, 2020.

[7] G. Peng, X. Huang, L. Zhou et al., "Solid-liquid two-phase flow and wear analysis in a large-scale centrifugal slurry pump," Engineering Failure Analysis, vol. 114, Article ID 104602, 2020.

[8] S. Tang, S. Yuan, and Y. Zhu, "Deep learning-based intelligent fault diagnosis methods toward rotating machinery," IEEE Access, vol. 8, no. 1, pp. 9335-9346, 2020.

[9] N. E. Huang, Z. Shen, and S. R. Long, "A new view OF nonlinear water waves: the Hilbert spectrum," Annual Review of Fluid Mechanics, vol. 31, no. 1, pp. 417-457, 1999.

[10] L. Yaguo, L. Jing, H. Zhengjia, and J. Z. Ming, "A review on empirical mode decomposition in fault diagnosis of rotating machinery," Mechanical Systems \& Signal Processing, vol. 35, no. 1-2, pp. 108-126, 2013.

[11] D. Yu, J. Cheng, and Y. Yang, "Application of EMD method and Hilbert spectrum to the fault diagnosis of roller bearings," Mechanical Systems and Signal Processing, vol. 19, no. 2, pp. 259-270, 2005.

[12] G. Liu, X. Hu, E. Wang et al., "SVR-EEMD: An Improved eemd method based on support vector regression extension in ppg signal denoising," Computational and Mathematical Methods In Medicine, vol. 2019, 10 pages, 2019.

[13] S. Xue, J. Tan, L. Shi et al., "Rope tension fault diagnosis in hoisting systems based on vibration signals using eemd, Improved Permutation Entropy, And PSO-SVM," Entropy, vol. 22, no. 2, 2020.

[14] Z. Hongmei, D. Chunlei, G. Senmao et al., "A quantified selfadaptive filtering method: effective IMFs selection based on CEEMD," Measurement Science \& Technology, vol. 29, no. 8, 2018.

[15] S. Hou and W. Guo, "Optimal denoising and feature extraction methods using modified ceemd combined with duffing system and their applications in fault line selection of non-solid-earthed network," Symmetry-base, vol. 12, no. 4, 2020. 
[16] H. Zhao, M. Sun, Wu Deng et al., "A new feature extraction method based on eemd and multi-scale fuzzy entropy for motor bearing," Entropy, vol. 19, no. 1, 2017.

[17] M. Li, H. Wang, G. Tang et al., "An improved method based on ceemd for fault diagnosis of rolling bearing," Advances in Mechanical Engineering, vol. 2014, Article ID 676205, 10 pages, 2014.

[18] L. Hongbin, W. Taiyong, and Y. Zhiqiang, "The quantitative research of interaction between key parameters and the effects on mechanical property in FDM," Advances in Materials Science and Engineering, vol. 2017, Article ID 9152954, 15 pages, 2017.

[19] H. Hu, K. He, T. Zhong et al., "Fault diagnosis of FDM process based on support vector machine (SVM)," Rapid Prototyping Journal, vol. 26, no. 2, pp. 330-348, 2020.

[20] C. Wang, L. Zhou, and Z. Li, "Survey of switch fault diagnosis for modular multilevel converter," IET Circuits, Devices \& Systems, vol. 13, no. 2, pp. 117-124, 2019.

[21] W. Yang, P. Norrlund, J. Bladh, J. Yang, and U. Lundin, "Hydraulic damping mechanism of low frequency oscillations in power systems: quantitative analysis using a nonlinear model of hydropower plants," Applied Energy, vol. 212, pp. 1138-1152, 2018.

[22] W. Zeng, S. A. Ismail, and E. Pappas, "Detecting the presence of anterior cruciate ligament deficiency based on a double pendulum model, intrinsic time-scale decomposition (ITD) and neural networks," Artificial Intelligence Review, vol. 53, no. 5, pp. 3231-3253, 2020.

[23] S. Zhang, J. Tian, A. Banerjee, and J. Li, “An efficient porcine acoustic signal denoising technique based on EEMD-ICAWTD," Mathematical Problems in Engineering, vol. 2019, no. 1, 12 pages, Article ID 2858740, 2019.

[24] H. Cui, Y. Qiao, Y. Yin, and M. Hong, "An investigation of rolling bearing early diagnosis based on high-frequency characteristics and self-adaptive wavelet de-noising," $\mathrm{Neu}$ rocomputing, vol. 216, pp. 649-656, 2016.

[25] Z. Qiao and Z. Pan, "SVD principle analysis and fault diagnosis for bearings based on the correlation coefficient," Measurement Science and Technology, vol. 26, no. 8, 2015.

[26] C. Liu, G. Cheng, X. Chen et al., "Planetary gears feature extraction and fault diagnosis method based on VMD and CNN," Sensors, vol. 18, no. 5, 2018.

[27] C. Gang, h. C. Xi, L. S. Xian, G. L. Hou, and F. Z. Chang, "A new method of gear fault diagnosis in strong noise based on multi-sensor information fusion," Journal of Vibration and Control, vol. 22, no. 6, pp. 1504-1515, 2016.

[28] J. FanZ. zhu et al., "Fault diagnosis of rotating machinery based on noise reduction using empirical mode decomposition and singular value decomposition," Journal of Vibroengineering, vol. 17, no. 1, pp. 164-174, 2015.

[29] H. Li, X. Qin, and Y. Sun, "K-SVD-based WVD enhancement algorithm for planetary gearbox fault diagnosis under a CNN framework," Measurement Ence and Technology, vol. 31, no. 2, 2020.

[30] Z. Li, B. Shi, X. Ren et al., "Research and application of weak fault diagnosis method based on asymmetric potential stochastic resonance," Measurement \& Control, vol. 52, no. 5-6, pp. 625-633, 2019.

[31] Z. H. Lai, S. B. Wang, G. Q. Zhang, C. L. Zhang, and J. W. Zhang, "Rolling bearing fault diagnosis based on adaptive multiparameter-adjusting bistable stochastic resonance," Shock and Vibration, vol. 2020, no. 9, pp. 1-15, 2020.
[32] R. Benzi, A. Sutera, and A. Vulpiani, "The mechanism of stochastic resonance," Journal of Physics A: Mathematical and General, vol. 14, no. 11, pp. L453-L457, 1981.

[33] Z. Li and B. Shi, "Research of fault diagnosis based on sensitive intrinsic mode function selection of eemd and adaptive stochastic resonance," Shock and Vibration, vol. 2016, no. 8, 12 pages, Article ID 2841249, 2016.

[34] Z. Li and B. Shi, "Fault diagnosis of rotating machinery based on stochastic resonance with a bistable confining potential," Shock and Vibration, vol. 2018, Article ID 8452509, 12 pages, 2018.

[35] W. Zhu, "Research on fault diagnosis and prediction and state evaluation methods of hydropower units," Doctoral dissertation Huazhong University of Science and Technology, Wuhan, China, 2016. 